\title{
Characteristics of a Saturated 18.9-nm Tabletop Laser Operating at 5-Hz Repetition Rate
}

\author{
M. A. Larotonda, B. M. Luther, Y. Wang, Y. Liu, D. Alessi, M. Berrill, A. Dummer, F. Brizuela, C. S. Menoni, \\ M. C. Marconi, V. N. Shlyaptsev, J. Dunn, and J. J. Rocca, Fellow, IEEE
}

Invited Paper

\begin{abstract}
We report the characteristics of a saturated high-repetition rate Ni-like Mo laser at $18.9 \mathrm{~nm}$. This table-top soft X-ray laser was pumped at a $5-\mathrm{Hz}$ repetition rate by 8 -ps 1 -J optical laser pulses impinging at grazing incidence into a precreated Mo plasma. The variation of the laser output intensity as a function of the grazing incidence angle of the main pump beam is reported. The maximum laser output intensity was observed for an angle of $20^{\circ}$, at which we measured a small signal gain of $65 \mathrm{~cm}^{-1}$ and a gain-length product $g \times l>15$. Spatial coherence measurements resulting from a Young's double-slit interference experiment show the equivalent incoherent source diameter is about $11 \mu \mathrm{m}$. The peak spectral brightness is estimated to be of the order of $1 \times 10^{24}$ photons $\mathrm{s}^{-1} \mathrm{~mm}^{-2} \mathrm{mrad}^{-2}$ within $0.01 \%$ spectral bandwidth. This type of practical, small scale, high-repetition soft X-ray laser is of interest for many applications.
\end{abstract}

Index Terms-Grazing incidence pumping, high-repetition rate, nickel-like Mo, X-ray laser.

\section{INTRODUCTION}

$\mathbf{T}$ HE DEVELOPMENT of practical small-scale sources of coherent soft X-ray radiation is of significant interest for numerous applications. Soft X-ray lasers operating in the gain-saturated regime can produce high-energy pulses of highly monochromatic coherent radiation at short wavelengths [1]. However, their widespread use in applications has been often limited by their very low-repetition rate of one shot every

Manuscript received August 31, 2004; revised September 20, 2004. This work was supported in part by the National Science Foundation Center for Extreme Ultraviolet Science and Technology under NSF Award EEC-0310717, with equipment developed under NSF Grant ECS-9977677, and by the W.M. Keck Foundation. Part of work was also performed under the auspices of the U.S. Dept. of Energy by the University of California Lawrence Livermore National Laboratory under Contract W-7405-Eng-48.

M. A. Larotonda, B. M. Luther, Y. Wang, D. Alessi, M. Berrill, A. Dummer, F. Brizuela, C. S. Menoni, M. C. Marconi, and J. J. Rocca are with the National Science Foundation ERC for Extreme Ultraviolet Science and Technology and the Electrical and Computer Engineering Department, Colorado State University, Fort Collins, CO 80523 USA (e-mail: mlaroton@engr.colostate.edu; bm1@lamar.colostate.edu; yong@engr.colostate.edu; rocca@engr.colostate.edu).

Y. Liu is with the National Science Foundation ERC for Extreme Ultraviolet Science and Technology and the Center for X-Ray Optics, Lawrence Berkeley National Laboratory, Berkeley, CA 94720 USA.

V. N. Shlyaptsev is with the Department of Applied Science, University of California Davis-Livermore, Livermore, CA 94551 USA.

J. Dunn is with the Lawrence Livermore National Laboratory, Livermore, CA 94550 USA.

Digital Object Identifier 10.1109/JSTQE.2004.838038 several minutes. High-repetition rate operation in the gain-saturated regime was first achieved at $46.9 \mathrm{~nm}$ in 1998 using capillary discharge excitation of an Ne-like Ar plasma at repetition rates up to $10 \mathrm{~Hz}$ [2]. Capillary discharge pumping allowed the utilization of small scale high-repetition rate soft X-ray lasers in numerous applications, including the determination of optical constants by reflectometry, material ablation studies, plasma interferometry, the characterization of soft X-ray optical components, and nanopatterning [3]. More recently, saturated optical field ionization lasers were reported to operate at a $10-\mathrm{Hz}$ repetition rate in the 41.8-nm line of Pd-like Xe [4] and in the 32.8-nm line of Ni-like $\mathrm{Kr}$ [5].

There is significant interest in extending the operation of high-repetition rate saturated lasers to shorter wavelengths. Several soft X-ray laser pumping configurations have been investigated to reduce the necessary laser pump energy with the goal of enabling both higher repetition rate operation and reducing the size and cost of the system [4]-[11]. Longitudinal pumping has produced nonsaturated amplification at $18.9 \mathrm{~nm}$ in Ni-like Mo using 10-Hz excitation [9], [10]. Excitation of a Mo target with $150-\mathrm{fs} 300-\mathrm{mJ}$ pulses impinging at $60^{\circ}$ from normal incidence resulted in the appearance of the 18.9- and 22.6-nm laser lines of Ni-like Mo [11]. Recently, it has been demonstrated that the energy deposition efficiency of the main heating pulse can be significantly increased by impinging at a grazing angle of incidence onto the target [12]-[15]. This inherently traveling wave pumping geometry takes advantage of the refraction of the pump beam to increase the interaction length of the rays in the gain region of the plasma [Fig. 1(a)], thereby increasing the fraction of the pump energy absorbed in that region. Pumping of the 18.9-nm line of Ni-like Mo at $14^{\circ}$ grazing incidence with $150 \mathrm{~mJ}$ of total pumping energy from a $10-\mathrm{Hz}$ laser was reported to generate a gain-length product of $\sim 15$ [14], and the use of 1-J heating pulses at the same angle of incidence resulted in operation in the gain-saturated regime with the generation of $\sim 150$-nJ soft X-ray laser output pulses [15].

Herein, we discuss the characteristics of a saturated 18.9-nm laser operating in the $4 \mathrm{~d}^{1} \mathrm{~S}_{0}-4 \mathrm{p}^{1} \mathrm{P}_{1}$ transition of Ni-like Mo operating at a repetition rate of $5 \mathrm{~Hz}$. The results were obtained at Colorado State University using a table-top Ti:sapphire pump laser system that generates a 120-ps $\sim 350-\mathrm{mJ}$ prepulse, and an 8-ps $\sim 1-\mathrm{J}$ main heating pulse. The experimental setup is 


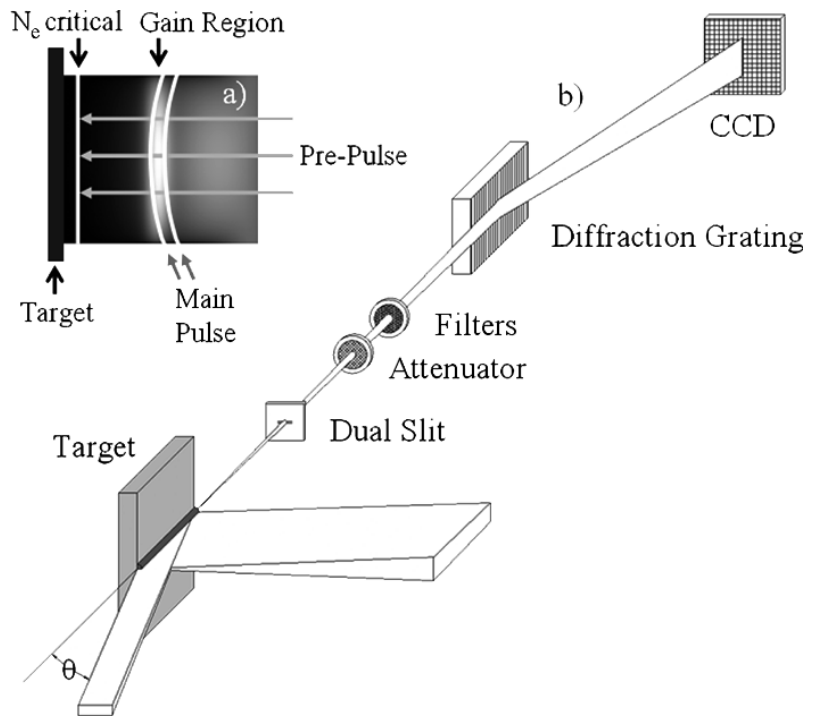

Fig. 1. (a) Schematic representation of the grazing incidence pumping configuration. (b) Schematic representation of the 18.9-nm laser setup, including the setup used to measure the spatial coherence.

described in Section II. The 18.9-nm soft X-ray laser output intensity was measured as a function of the grazing angles of incidence between 14 and $26^{\circ}$. The gain and spatial coherence were subsequently measured for the angle at which the highest intensity was observed, $20^{\circ}$. Section III reports the variation of the laser output intensity as a function of the grazing incidence angle and the measurement of the gain at a grazing angle of $20^{\circ}$. Data that illustrates the operation of the laser at $5-\mathrm{Hz}$ repetition rate is also shown in this section. The measurement of the spatial coherence is discussed in Section IV.

\section{LASER CONFIGURATION}

The soft X-ray laser was pumped with a 120-ps prepulse followed by an 8-ps short pulse produced by the same Ti:sapphire laser operating at a center wavelength of $800 \mathrm{~nm}$. The Ti:sapphire pump laser consists of a mode-locked oscillator and three stages of chirped-pulse amplification. The nanojoule pulses from the laser oscillator are stretched and subsequently amplified in a chain of three Ti:sapphire amplifiers. Eight passes thought the first stage amplifier increases the pulse energy to about $2 \mathrm{~mJ}$. Further amplification in a five-pass second stage bow-tie amplifier further increases the pulse energy to about $200 \mathrm{~mJ}$. The output of the second stage amplifier is spatially filtered and injected into a final three-pass amplifier that is pumped by a 5-J doubled Nd-YAG laser. This final amplification stage increases the $800-\mathrm{nm}$ pulse energy to about $2 \mathrm{~J}$. A multilayer coated beam splitter was placed at the output of the third-stage amplifier to direct about $20 \%$ of the uncompressed laser energy to a prepulse arm. The rest of the third-stage output was sent to a vacuum grating compressor, where it was compressed to about 8 ps. Amplification of spurious light from the first stage amplifier produced a pulse with a few percent of the total energy that preceded the main pulse by $6.7 \mathrm{~ns}$. The repetition rate of the soft X-ray laser in these experiments was set by the $5-\mathrm{Hz}$ repetition frequency of the third-stage amplifier but could be increased up to $10 \mathrm{~Hz}$. An electromechanical shutter was placed on the pump beam of the first amplifier to allow for single shot data acquisition in some of the measurements.

The pumping geometry is schematically illustrated in Fig. 1(b). A line focus plasma was first formed by irradiating a 2-mm-thick 4-mm-wide polished Mo slab target at near normal incidence with an intensity of $2.4 \times 10^{12} \mathrm{~W} \mathrm{~cm}^{-2}$ obtained by focusing the long duration prepulse into a $4.1 \mathrm{~mm}$ full-width at half-maximum (FWHM) long $\times 30 \mu \mathrm{m}$ FWHM wide line. The line focus was formed using the combination of an $\mathrm{f}=67.5 \mathrm{~cm}$ spherical lens and an $\mathrm{f}=200 \mathrm{~cm}$ cylindrical lens. The plasma created by this prepulse was allowed to expand for a selected time delay and was then rapidly heated by the 8-ps FWHM duration pulse impinging at a selected grazing incidence angle. The short heating pulse was focused into a 30- $\mu$ m FWHM wide line using a $\mathrm{f}=76.2 \mathrm{~cm}$ focal length multilayer-coated parabolic mirror placed at $7^{\circ}$ from normal incidence. The surface of the target was positioned to form selected grazing incidence angles of either $14^{\circ}, 20^{\circ}$, or $26^{\circ}$ with respect to the axis of the short pulse beam. The off-axis placement of the paraboloid formed an astigmatic focus that resulted in a line that was further elongated to $4.1 \mathrm{~mm}$ when intercepted at grazing incidence by the target. The overlap of the two line foci on target was monitored and adjusted by imaging the target with a $\mathrm{f}=25 \mathrm{~cm}$ lens onto a charge-coupled device (CCD). The delay between the long laser prepulse and the short grazing incidence pulse was adjusted utilizing a variable delay stage and was monitored with a semiconductor photodiode displayed on a 1-GHz bandwidth digitizing oscilloscope.

The plasma emission was monitored using a flat field spectrograph composed of a $1200 \mathrm{l} / \mathrm{mm}$ gold-coated variably spaced spherical grating placed at $87^{\circ}$ and a $2048 \times 2048$ pixel, 1 square in, back-illuminated CCD detector array placed in the image plane of the grating $48 \mathrm{~cm}$ from the target. The plasma radiation was filtered with a combination of $\mathrm{Zr}$ and $\mathrm{Al}$ filters, selected to facilitate the study of the 18.9-nm line of Ni-like Mo. The use of the $\mathrm{Zr}$ filter precluded the observation of the 22.6-nm line of Ni-like Mo recently reported using this configuration [13]. A set of metallic meshes of measured transmissivity were positioned between the target and the grating to further attenuate the beam with the purpose of avoiding saturation of the CCD detector by the soft X-ray laser beam. When more that one mesh was used, care was taken to avoid the formation of Moiré patterns that can cause unacceptable spatial variations of the transmissivity.

\section{LASER CHARACTERISTICS AND DEMONSTRATION OF 5-Hz OPERATION}

The selection of the angle of incidence that maximizes the pump beam energy deposition in the gain region is key to improving the soft X-ray laser efficiency. Fig. 2 shows the measured variation of the intensity of the $18.9-\mathrm{nm}$ line for main heating pulse grazing incidence angles of $14^{\circ}, 20^{\circ}$, and $26^{\circ}$. The data was obtained using $\sim 0.35 \mathrm{~J}$ of prepulse energy and about $1 \mathrm{~J}$ of short pulse energy impinging on 4-mm-long Mo targets. For each angle the time delay between the prepulse and the short pulse was optimized. At an incidence angle of $14^{\circ}$ large amplification and strong lasing were observed. However, at this 


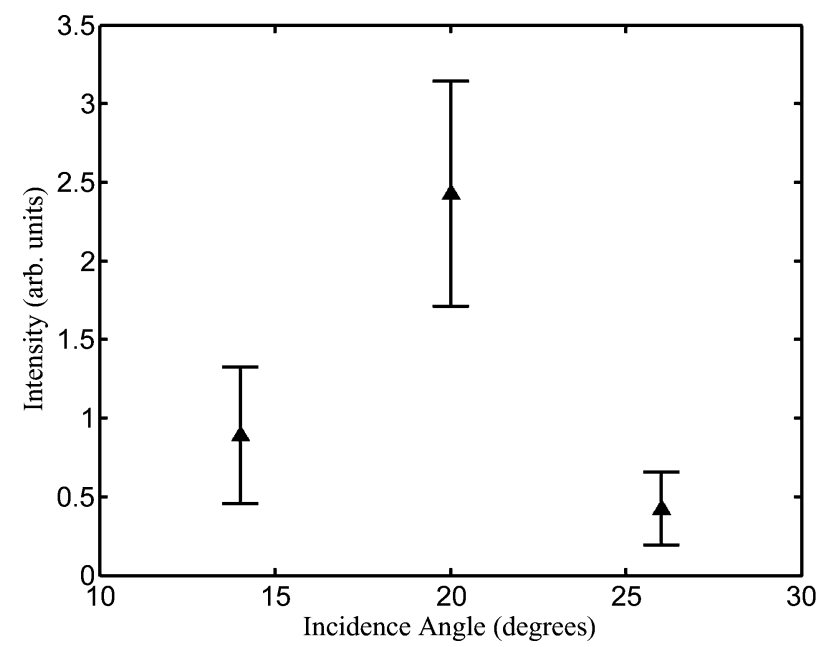

Fig. 2. Variation of the intensity of the 18.9-nm Ni-like Mo laser beam as a function of angle of incidence of the main heating pulse measured with respect to the target surface. Data was obtained using a 0.35-J 120-ps duration prepulse followed by 1-J 8-ps duration grazing incidence short pulse separated by a delay of $500 \mathrm{ps}$.

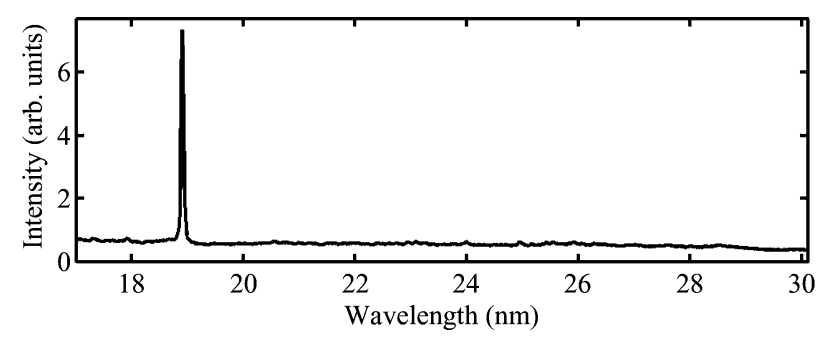

Fig. 3. On-axis spectrum of a 4-mm plasma showing strong lasing in the 18.9-nm line of Ni-like Mo.

angle the pump beam energy is deposited in a region where the electron density is slightly lower than the optimum value for maximum output intensity. The output intensity was observed to increase for an angle of $20^{\circ}$, for which refraction helps to couple the pump beam into a region of higher electron density. This angle of refraction corresponds to an electron density of $2 \times 10^{20} \mathrm{~cm}^{-3}$. Strong lasing was observed to occur over a wide range of time delays, ranging from about 300 to 1100 ps, values for which the laser output intensity is half the value measured at the optimum delay of $500 \mathrm{ps}$. An on-axis spectrum is shown in Fig. 3. The 18.9-nm laser line completely dominates the spectrum. A further increase of the angle to $26^{\circ}$ caused a significant decrease of the soft X-ray laser intensity. At this steeper angle of incidence, a significant fraction of the beam energy is absorbed in a higher density region, where the electron density gradients are too steep for optimum amplification. Also contributing to a lower laser output intensity at this angle is the shorter duration of the gain and the increased mismatch between the velocity of the traveling wave of the pump and the speed of light in the plasma.

Fig. 4 shows the variation of the 18.9-nm laser line intensity as a function of target length for the most favorable of the angles studied, $20^{\circ}$. Each point is the average of about ten laser shots and the error bars correspond to plus-minus one standard deviation. The data was fit with an analytic expression for the output laser intensity taking into account gain saturation [16]. The resulting gain coefficient is $65 \mathrm{~cm}^{-1}$, slightly larger than the value

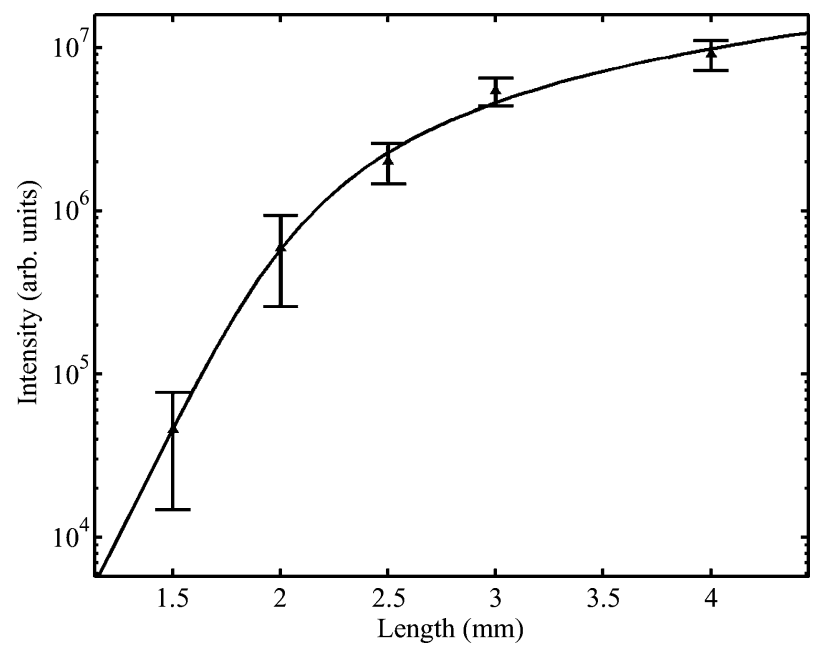

Fig. 4. Variation of the intensity of the 18.2-nm line of Ni-like Mo as a function of target length. Best fit of the data with an analytical expression that relates the gain and the output laser intensity taking into account gain saturation yields a small signal gain coefficient of $65 \mathrm{~cm}^{-1}$ and a gain-length product $g \times l=15.3$.

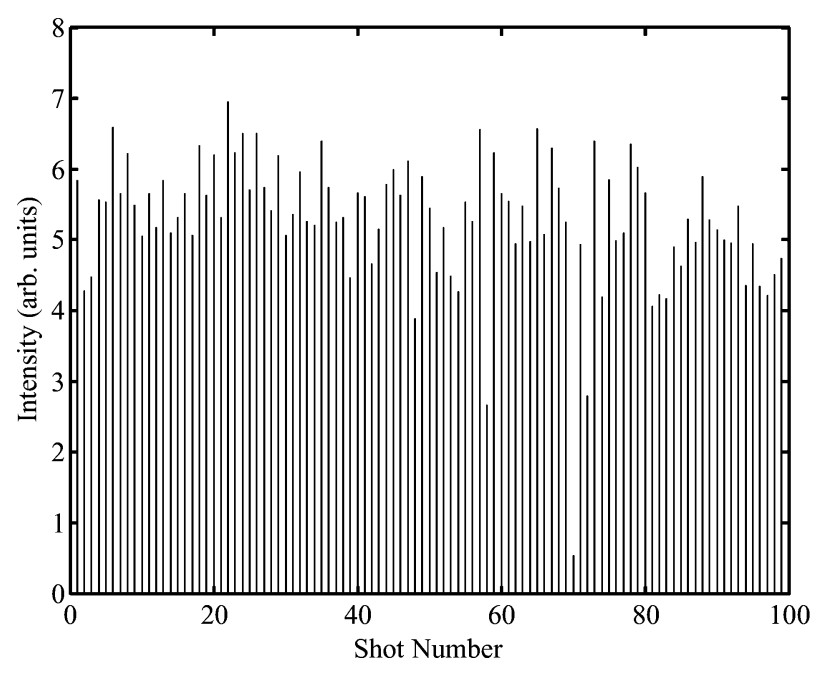

Fig. 5. Intensity of 100 consecutive 18.9-nm laser shots acquired at a repetition rate of $5 \mathrm{~Hz}$.

previously measured for $14^{\circ}$ grazing incidence pumping in the same setup [14]. The gain-length product reaches $g \times l \sim 15.3$. Such amplification is known to produce gain saturation in most collisionally excited soft X-ray lasers. Soft X-ray laser operation in the gain-saturated regime is highly desirable to enhance the laser output efficiency and reduce shot-to-shot variations of the intensity. The 18.9-nm laser output pulse energy was estimated integrating the CCD counts taking into account the losses and the quantum efficiency of the detector. The laser pulse energy for the best shots corresponding to the 4-mm-long targets is estimated to be about $250 \mathrm{~nJ}$, which at a repetition rate of $5 \mathrm{~Hz}$ amounts to an average power of about $1 \mu \mathrm{W}$. The beam divergence in the direction parallel to the target surface was measured to be about 9 milliradians.

Operation of the 18.9-nm laser at a repetition rate of $5 \mathrm{~Hz}$ is illustrated in Fig. 5 for a series of 100 consecutive shots. The laser line intensity for every shot was recorded with the CCD using pixel binning to increase read-out speed. To enhance the shot-to-shot reproducibility the target was moved at a velocity of 
$0.2 \mathrm{~mm} / \mathrm{s}$. Lasing is observed for all shots, with an intensity variation characterized by a standard deviation of $17 \%$ of the mean. Soft X-ray laser operation at a $10-\mathrm{Hz}$ repetition rate should be readily achieved with the same setup by making relatively minor adjustments to the pump laser.

\section{Spatial CohEREnCE MEASUREMENTS}

The spatial coherence of the $18.9-\mathrm{nm}$ radiation produced at $20^{\circ}$ grazing incidence pumping was characterized by placing double slits $5.5 \mathrm{~cm}$ from the Mo target. The laser beam profile at this location was found to have a width (FWHM) of approximately $500 \mu \mathrm{m}$. The double slits were prepared by laser drilling two 4-mm-long 5- $\mu$ m-wide slits on 12.5 - $\mu$ m-thick stainless steel substrates (National Aperture Inc., NH). Slit separations of 50 and $75 \mu \mathrm{m}$ were used in the experiments. The grating spectrometer mentioned in Section II was used to reduce the background plasma radiation and increase the signal-to-noise ratio of the recorded interference pattern [see Fig. 1(b)]. The slits were placed horizontally, perpendicular to the grating groove direction, introducing diffraction and interference in the vertical direction. The length of the slits $(4 \mathrm{~mm})$ was much longer than the laser beam diameter; therefore, the slits had minimal effect on the spectral line formation. Modulation was observed along the length of the 18.9-nm laser line, indicating the degree of spatial coherence between light illuminating each of the two individual slits. As shown in Fig. 6(a), this method produced a very clear interference pattern on the 18.9-nm laser line with a single laser shot, even though the slits blocked $>90 \%$ of the photons. In fact, the gain-saturated operation of the laser produced such a high photon flux level that the first-order spectral line of the 18.9-nm radiation saturated the $\mathrm{CCD}$. Therefore, to measure the fringe visibility we used the line produced by the second order of the diffraction grating.

The evaluation of the degree of spatial coherence using the double-pinhole or the double-slit technique follows the description of partially coherent radiation in terms of the mutual intensity and the complex coherence factor [17]. In an ideal double slit experiment where both slits are equally illuminated, the modulus of the complex coherence factor is equal to the fringe visibility, defined as $\mathrm{V}=\left(\mathrm{I}_{\max }-\mathrm{I}_{\min }\right) /\left(\mathrm{I}_{\max }+\mathrm{I}_{\min }\right)$, where $I_{\max }$ and $I_{\min }$ are the maximum and minimum intensities of the fringe pattern. Fig. 6(a) shows the interference generated on the 18.9-nm laser line by a double slit with $50-\mu \mathrm{m}$ separation. The horizontal lineouts for 50- and $75-\mu \mathrm{m}$ slit separations are shown in Fig. 6(b) and (c), respectively. From the fringe visibilities, the degree of spatial coherence is determined to be 0.4 between two slits separated by $50 \mu \mathrm{m}$ and 0.1 for $75-\mu \mathrm{m}$ separation.

Using the van Cittert-Zernike theorem [17] a near-Gaussian intensity profile, incoherent source of roughly $11-\mu \mathrm{m}$ diameter (FWHM) would be needed to produce similar fringe visibilities with the same slit pairs at the same distance $(5.5 \mathrm{~cm})$ from the source. This is about $3 \times$ smaller than the $30-\mu \mathrm{m}$ pump laser beam width. An additional characteristic of a pulsed light source closely related to its coherence properties is the peak spectral brightness. With a measured divergence angle of about 9 milliradians and assuming the pulse duration is approximately the
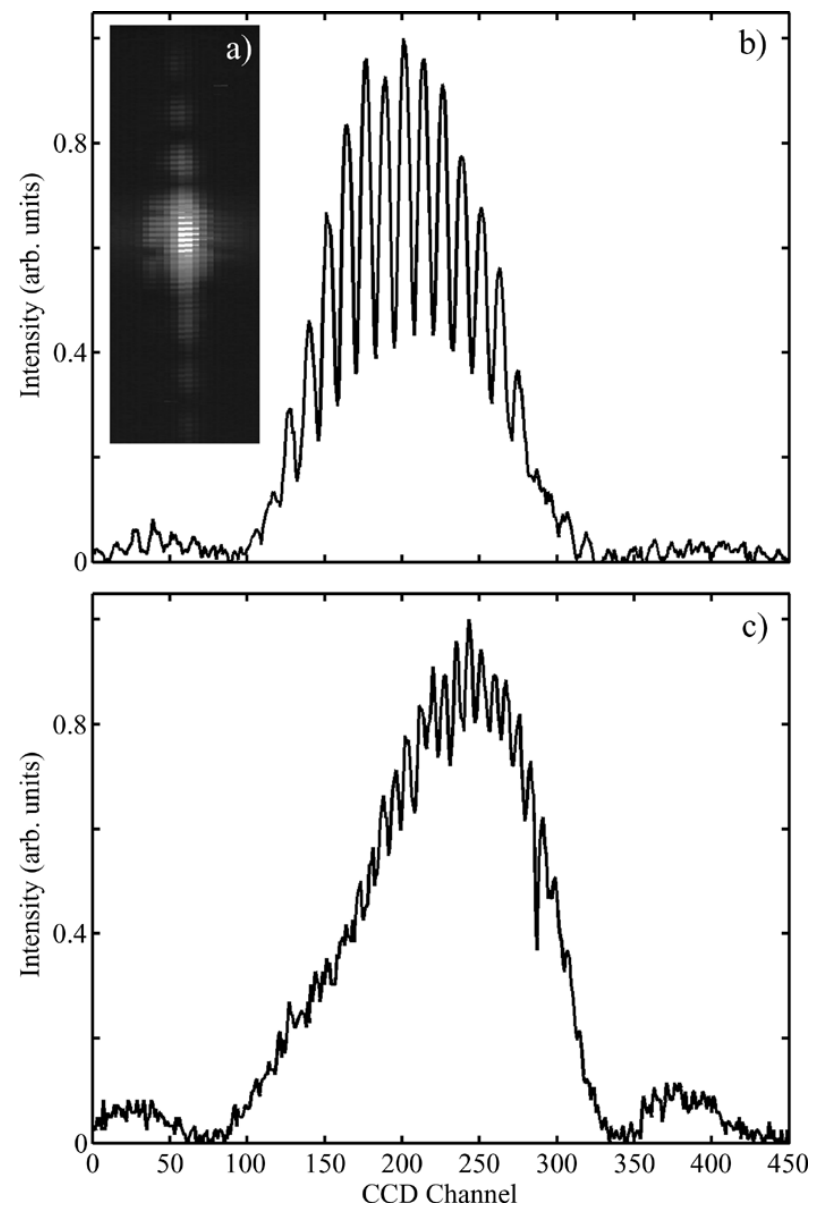

Fig. 6. (a) Interferogram obtained placing a slit pair with $50-\mu \mathrm{m}$ separation at $5.5 \mathrm{~cm}$ from the exit of a 4-mm-long Mo target. (b) Lineout for the $50-\mu \mathrm{m}$ separation slit pair obtained integrating the interference pattern in the direction perpendicular to the line. (c) Lineout for the slit pair with $75-\mu \mathrm{m}$ separation. Asymmetry is due to the uneven illumination of the slit pair that was not centered on the beam axis.

duration of the pump pulse divided by $(g \times l)^{1 / 2}, \sim 2 \mathrm{ps}$, a reasonable approximation for a nonstrongly saturated laser, the peak spectral brightness of this source is estimated to be of the order of $1 \times 10^{24}$ in units of photons s${ }^{-1} \mathrm{~mm}^{-2} \mathrm{mrad}^{-2}$ within $0.01 \%$ spectral bandwidth.

\section{CONCLUSION}

In conclusion, we have demonstrated $5-\mathrm{Hz}$ repetition rate operation of a saturated transient Ni-like Mo 18.9-nm laser using grazing incidence pumping at three angles between $14^{\circ}$ and $26^{\circ}$ and have measured its characteristics. The results could be readily extended to $10-\mathrm{Hz}$ repetition rate with minor modifications to the same table-top pump laser. For the pumping conditions investigated the maximum laser output intensity was observed at a grazing incidence pump angle of $20^{\circ}$. Spatial coherence measurements conducted performing a Young's double-slit experiment show an equivalent incoherent source of $11-\mu \mathrm{m}$ diameter (FWHM). The peak spectral brightness of this laser is estimated to be of the order of $1 \times 10^{24}$ photons $\mathrm{s}^{-1} \mathrm{~mm}^{-2} \mathrm{mrad}^{-2}$, within $0.01 \%$ spectral bandwidth. These bright, small scale, high-repetition soft X-ray lasers are of interest for many applications. 


\section{ACKNOWLEDGMENT}

The authors would like to thank A. Weith for her assistance.

\section{REFERENCES}

[1] G. J. Tallents, "The physics of soft X-ray lasers pumped by electron collisions in laser plasmas," J. Phys. D, Appl. Phys., vol. 36, pp. R259-R276, 2003.

[2] B. R. Benware, C. D. Macchietto, C. H. Moreno, and J. J. Rocca, "Demonstration of a high average power tabletop soft X-ray laser," Phys. Rev. Lett., vol. 81, pp. 5804-5806, 1998.

[3] J. J. Rocca, M. Frati, B. Benware, M. Seminario, J. Filevich, M. Marconi, K. Kanizay, A. Ozols, I. A. Artiukov, A. Vinogradov, and Y. A. Uspenskii, "Capillary discharge tabletop soft X-ray lasers reach new wavelengths and applications," C. R. Acad. Sci. Paris, vol. 1, pp. 1065-1081, 2000

[4] S. Sebban, R. Haroutunian, P. Balcou, G. Grillon, A. Rousse, S. Kazamias, T. Marin, J. P. Rousseau, L. Notebaert, M. Pittman, J. P. Chambaret, A. Antonetti, D. Hulin, D. Ros, A. Klisnick, A. Carillon, P. Jaegle, G. Jamelot, and J. F. Wyart, "Saturated amplification of a collisionally pumped optical-field-ionization soft X-ray laser at $41.8 \mathrm{~nm}$," Phys. Rev. Lett., vol. 86, pp. 3004-3007, 2001.

[5] S. Sebban, T. Mocek, D. Ros, L. Upcraft, P. Balcou, R. Haroutunian, G. Grillon, B. Rus, A. Klisnick, A. Carillon, G. Jamelot, C. Valentin, A. Rousse, J. P. Rousseau, L. Notebaert, M. Pittman, and D. Hulin, "Demonstration of a Ni-like $\mathrm{Kr}$ optical-field-ionization collisional soft X-ray laser at 32.8 nm," Phys. Rev. Lett., vol. 89, 2002.

[6] K. A. Janulewicz, A. Lucianetti, G. Priebe, W. Sandner, and P. V. Nickles, "Saturated Ni-like Ag X-ray laser at $13.9 \mathrm{~nm}$ pumped by a single picosecond laser pulse," Phys. Rev. A, vol. 68, 2003.

[7] S. Basu, J. Goodberlet, M. Muendel, S. Kaushik, and P. Hagelstein, "Spectral measurements of Ni-like Mo plasmas," in Proc. Inst. Phys. Conf. Series, vol. 125, 1992, pp. 71-74.

[8] D. V. Korobkin, C. H. Nam, S. Suckewer, and A. Goltsov, "Demonstration of soft X-ray lasing to ground state in Li III," Phys. Rev. Lett., vol. 77, pp. 5206-5209, 1996.

[9] T. Ozaki, R. A. Ganeev, A. Ishizawa, T. Kanai, and H. Kuroda, "Highly directive $18.9 \mathrm{~nm}$ nickel-like molybdenum X-ray laser operating at 150 mJ pump energy," Phys. Rev. Lett., vol. 89, 2002.

[10] R. Li and Z.Z. Xu, "High efficiency transient collisional excitation X-ray laser in Ni-like Mo ions," J. Physique IV, vol. 11 (PR2), pp. 27-35, 2001.

[11] R. Tommasini, J. Nilsen, and E. E. Fill, "Investigations on $10-\mathrm{Hz}$ subJoule fs-laser pumped neon- and nickel-like X-ray lasers," in Proc. SPIE, vol. 4505, 2001, pp. 85-92.

[12] V. N. Shlyaptsev, J. Dunn, S. Moon, R. Smith, R. Keenan, J. Nilsen, K. B. Fournier, J. Kuba, A. L. Osterheld, J. J. Rocca, B. Luther, Y. Wang, and M. Marconi, "Numerical studies of transient and capillary X-ray lasers and their applications," in Proc. SPIE, vol. 5197, 2003, pp. 221-228.

[13] R. Keenan, J. Dunn, V. N. Shlyaptsev, R. Smith, P. K. Patel, and D. F. Price, "Efficient pumping schemes for high average brightness collisional X-ray lasers," in Proc. SPIE, vol. 5197, 2003, pp. 213-220.

[14] R. Keenan, J. Dunn, P. K. Patel, D. F. Price, R. F. Smith, and V. N. Shlyaptsev, "High repetition rate grazing incidence pumped X-ray laser operating at 18.9 nm," Phys. Rev. Lett., 2004.

[15] B. M. Luther, Y. Wang, M. A. Larotonda, D. Alessi, M. Berrill, M. Marconi, V. N. Shlyaptsev, and J. J. Rocca, "Saturated $18.9 \mathrm{~nm}$ laser in a $\mathrm{Ni}$-like Mo plasma excited by a high repetition rate table-top laser," $O p t$. Lett., 2004.

[16] G. J. Tallents, Y. Abou-Ali, M. Edwards, R. E. King, G. J. Pert, S. J. Pestehe, F. Strati, R. Keenan, C. L. S. Lewis, S. Topping, O. Guilbaud, A. Klisnick, D. Ros, R. Clarke, D. Neely, and M. Notley, "Saturated and short pulse duration X-ray lasers," in AIP Proc. X-ray Lasers 2002: 8th Int. Conf. X-ray Lasers, vol. 641, 2002, pp. 291-298.

[17] M. Born and E. Wolf, Principles of Optics, 7th ed. Cambridge, U.K.: Cambridge Univ. Press, 1999, ch. X.
M. A. Larotonda, photograph and biography not available at the time of publication.

B. M. Luther, photograph and biography not available at the time of publication.

Y. Wang, photograph and biography not available at the time of publication.

Y. Liu, photograph and biography not available at the time of publication.

D. Alessi, photograph and biography not available at the time of publication.

M. Berrill, photograph and biography not available at the time of publication.

A. Dummer, photograph and biography not available at the time of publication

F. Brizuela, photograph and biography not available at the time of publication.

C. S. Menoni, photograph and biography not available at the time of publication.

M. C. Marconi, photograph and biography not available at the time of publication.

V. N. Shlyaptsev, photograph and biography not available at the time of publication.

J. Dunn, photograph and biography not available at the time of publication.

J. J. Rocca, photograph and biography not available at the time of publication 